Supporting information for

\title{
Determinant Factor for Thermodynamic Stability of Sulfuric Acid-Amine Complexes
}

Jia Han, Lei Wang, Hanhui Zhang, Quyan Su, Xiaoguo Zhou, ${ }^{*}$ Shilin Liu*

Hefei National Laboratory for Physical Sciences at the Microscale, Department of

Chemical Physics, University of Science and Technology of China, Hefei, Anhui 230026,

P. R. China

\section{Contents:}

Figure S1. Optimized geometries of SA-EA complexes, calculated at the B3LYP-D3(BJ)/6-311++(3df,3pd) level of theory.

Figure S2. Optimized geometries of SA-PPA complexes, calculated at the B3LYP-D3(BJ)/6-311++(3df,3pd) level of theory.

Figure S3. Optimized geometries of SA-IPA complexes, calculated at the B3LYP-D3(BJ)/6-311++(3df,3pd) level of theory.

Figure S4. Optimized geometries of SA-tBA complexes, calculated at the B3LYP-D3(BJ)/6-311++(3df,3pd) level of theory.

Figure S5. Optimized geometries of SA-DMA complexes, calculated at the B3LYP-D3(BJ)/6-311++(3df,3pd) level of theory.

Figure S6. Optimized geometries of SA-EMA complexes, calculated at the B3LYP-D3(BJ)/6-311++(3df,3pd) level of theory.

Figure S7. Optimized geometries of SA-TMA complexes, calculated at the B3LYP-D3(BJ)/6-311++(3df,3pd) level of theory.

Figure S8. Optimized geometries of sulfuric acid-amine complexes, calculated at the M06-2X-D3/6-311++(3df,3pd) and $\omega \mathrm{B} 97 \mathrm{XD} / 6-311++(3 \mathrm{df}, 3 \mathrm{pd})$ level of theory.

Table S1. Gas-phase basicities and proton affinities of amines. ${ }^{1}$

Table S2. ZPE-corrected electronic energies, enthalpies, and Gibbs free energy changes for the formation of sulfuric acid-amine complexes at $298.15 \mathrm{~K}$ calculated at the M06-2X-D3/6-311++(3df,3pd) and $\omega \mathrm{B} 97 \mathrm{XD} / 6-311++(3 \mathrm{df}, 3 \mathrm{pd})$ level of theory. 


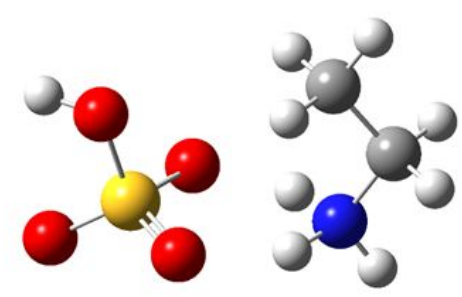

Iso $\mathrm{A}, \Delta \mathrm{E}=0.00 \mathrm{kcal} / \mathrm{mol}$

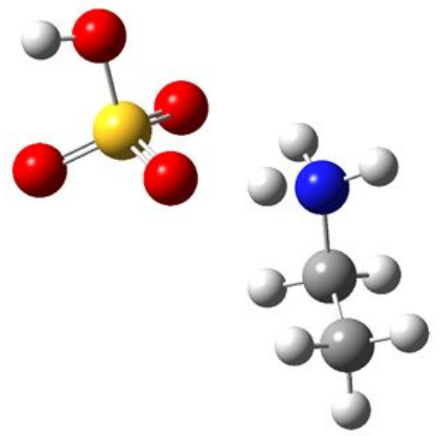

Iso $\mathrm{C}, \Delta \mathrm{E}=0.47 \mathrm{kcal} / \mathrm{mol}$

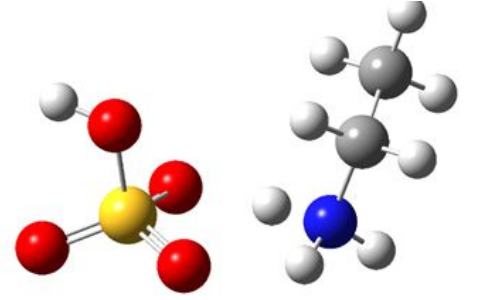

Iso $\mathrm{B}, \Delta \mathrm{E}=0.36 \mathrm{kcal} / \mathrm{mol}$

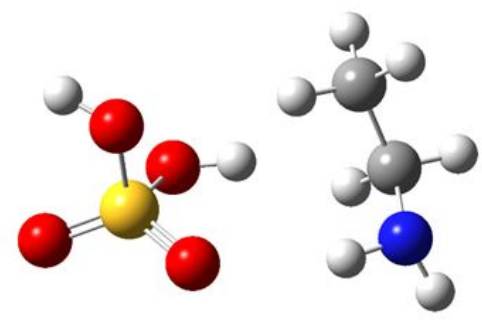

Iso $\mathrm{D}, \Delta \mathrm{E}=16.76 \mathrm{kcal} / \mathrm{mol}$

Figure S1. Optimized geometries of SA-EA complexes, calculated at the B3LYP-D3(BJ)/6-311++(3df,3pd) level of theory.

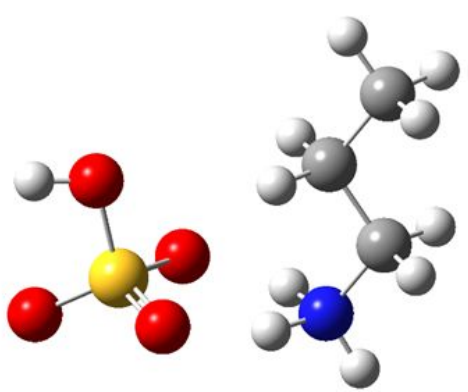

Iso $\mathrm{A}, \Delta \mathrm{E}=0.00 \mathrm{kcal} / \mathrm{mol}$

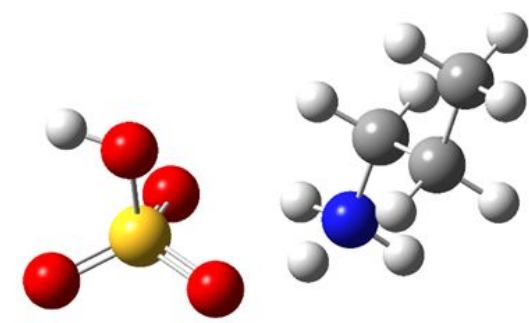

Iso $\mathrm{C}, \Delta \mathrm{E}=0.47 \mathrm{kcal} / \mathrm{mol}$

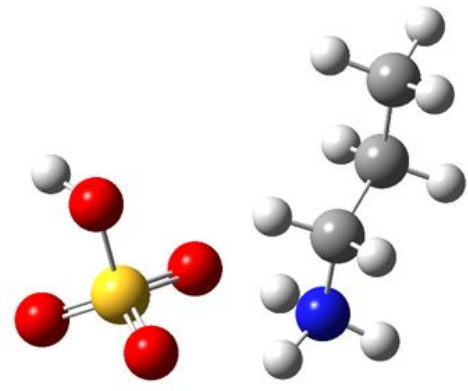

Iso $\mathrm{B}, \Delta \mathrm{E}=0.37 \mathrm{kcal} / \mathrm{mol}$

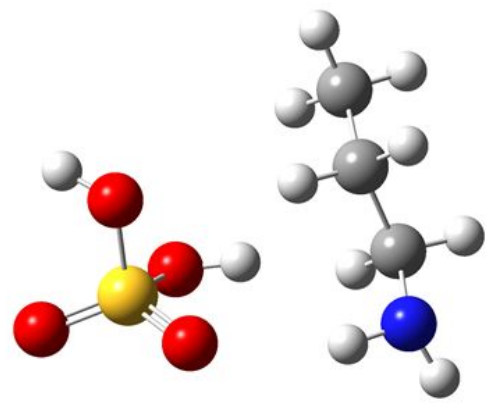

Iso $\mathrm{D}, \Delta \mathrm{E}=16.62 \mathrm{kcal} / \mathrm{mol}$

Figure S2. Optimized geometries of SA-PPA complexes, calculated at the B3LYP-D3(BJ)/6-311++(3df,3pd) level of theory. 


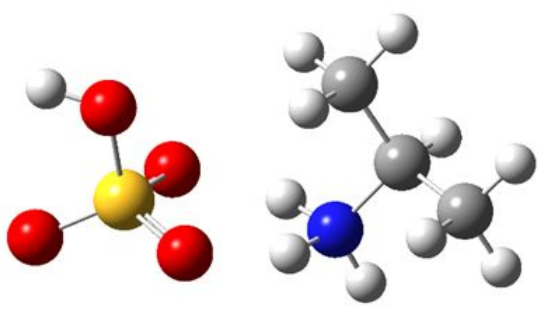

Iso $\mathrm{A}, \Delta \mathrm{E}=0.00 \mathrm{kcal} / \mathrm{mol}$

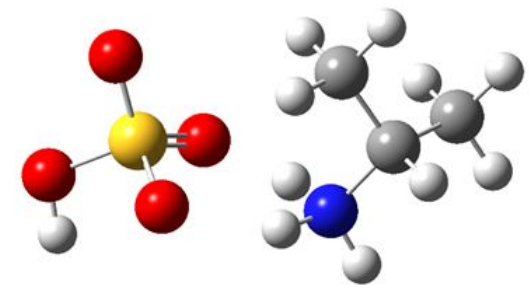

Iso $\mathrm{C}, \Delta \mathrm{E}=1.44 \mathrm{kcal} / \mathrm{mol}$

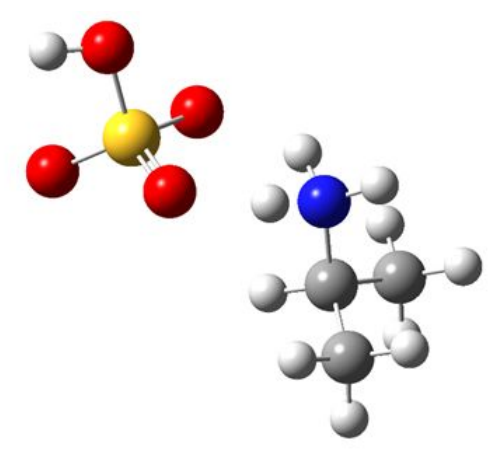

Iso $\mathrm{B}, \Delta \mathrm{E}=0.33 \mathrm{kcal} / \mathrm{mol}$

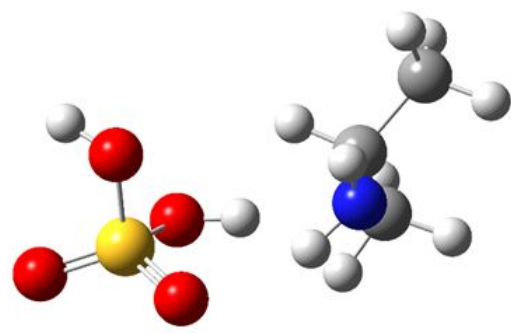

Iso $\mathrm{D}, \Delta \mathrm{E}=17.23 \mathrm{kcal} / \mathrm{mol}$

Figure S3. Optimized geometries of SA-IPA complexes, calculated at the B3LYP-D3(BJ)/6-311++(3df,3pd) level of theory.
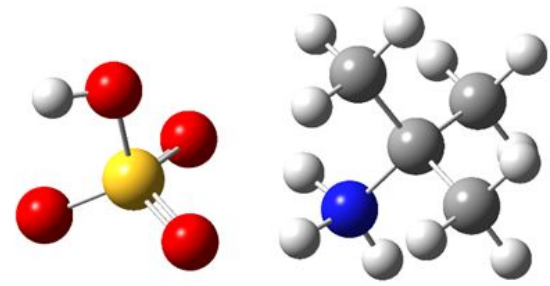

Iso $\mathrm{A}, \Delta \mathrm{E}=0.00 \mathrm{kcal} / \mathrm{mol}$

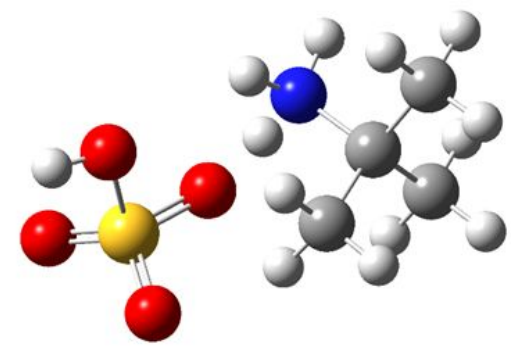

Iso $\mathrm{C}, \Delta \mathrm{E}=3.37 \mathrm{kcal} / \mathrm{mol}$

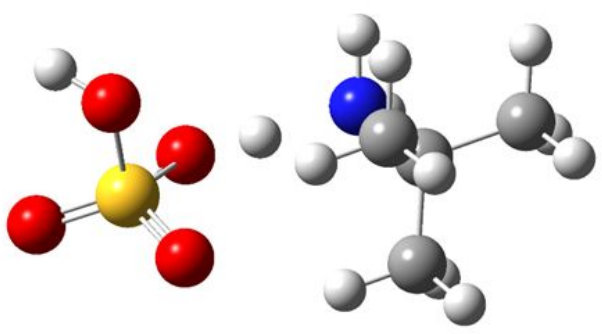

Iso $\mathrm{B}, \Delta \mathrm{E}=2.88 \mathrm{kcal} / \mathrm{mol}$
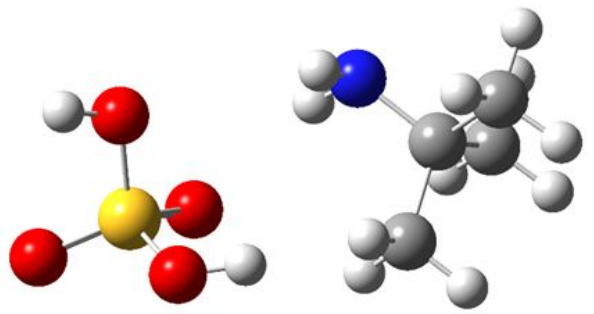

Iso $\mathrm{D}, \Delta \mathrm{E}=18.38 \mathrm{kcal} / \mathrm{mol}$

Figure S4. Optimized geometries of SA-tBA complexes, calculated at the B3LYP-D3(BJ)/6-311++(3df,3pd) level of theory. 


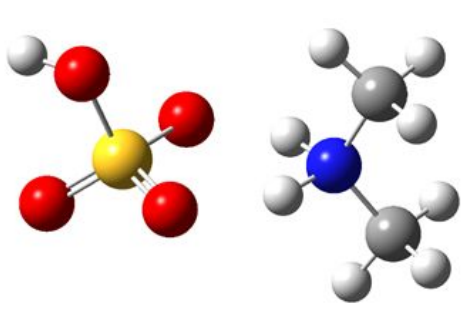

Iso $\mathrm{A}, \Delta \mathrm{E}=0.00 \mathrm{kcal} / \mathrm{mol}$

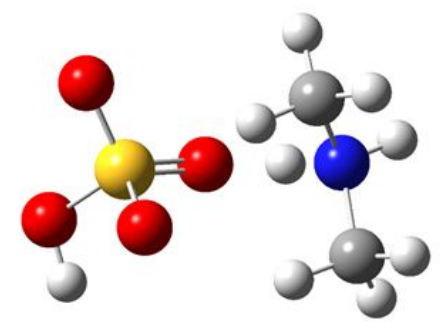

Iso $\mathrm{C}, \Delta \mathrm{E}=2.67 \mathrm{kcal} / \mathrm{mol}$

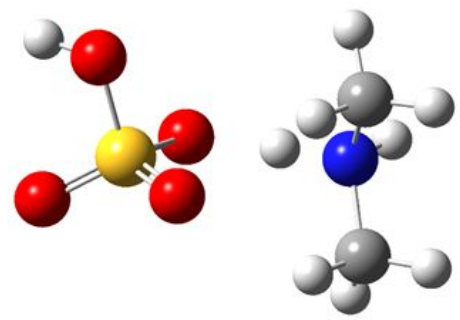

Iso $\mathrm{B}, \Delta \mathrm{E}=1.05 \mathrm{kcal} / \mathrm{mol}$

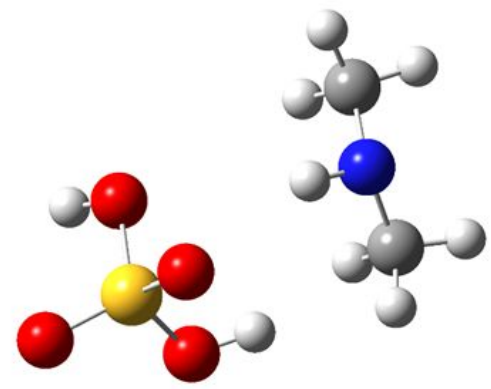

Iso $\mathrm{D}, \Delta \mathrm{E}=18.87 \mathrm{kcal} / \mathrm{mol}$

Figure S5. Optimized geometries of SA-DMA complexes, calculated at the B3LYP-D3(BJ)/6-311++(3df,3pd) level of theory.

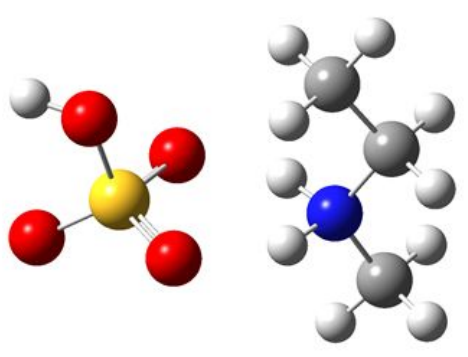

Iso $\mathrm{A}, \Delta \mathrm{E}=0.00 \mathrm{kcal} / \mathrm{mol}$

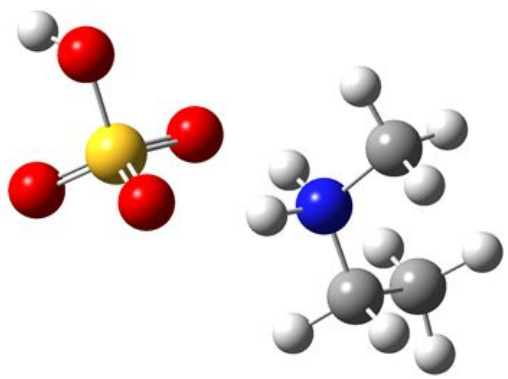

Iso $\mathrm{C}, \Delta \mathrm{E}=1.29 \mathrm{kcal} / \mathrm{mol}$

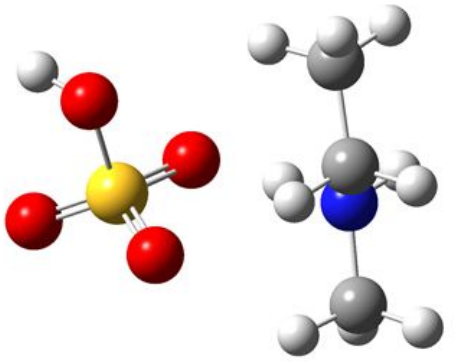

Iso $\mathrm{B}, \Delta \mathrm{E}=0.97 \mathrm{kcal} / \mathrm{mol}$

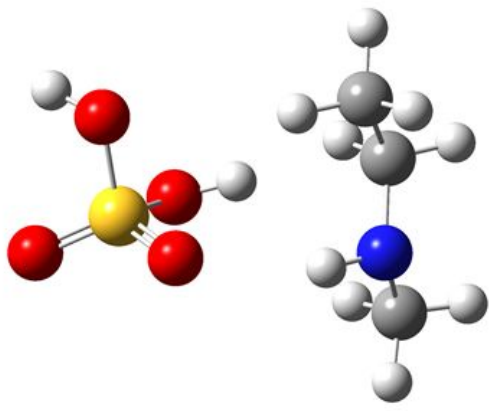

Iso $\mathrm{D}, \Delta \mathrm{E}=19.76 \mathrm{kcal} / \mathrm{mol}$

Figure S6. Optimized geometries of SA-EMA complexes, calculated at the B3LYP-D3(BJ)/6-311++(3df,3pd) level of theory. 


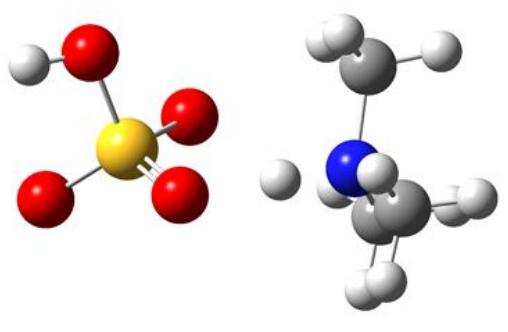

Iso $\mathrm{A}, \Delta \mathrm{E}=0.00 \mathrm{kcal} / \mathrm{mol}$

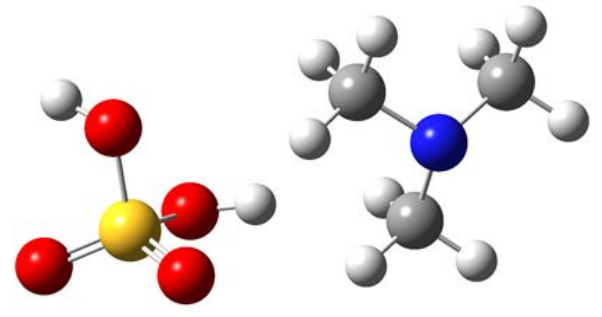

Iso $\mathrm{C}, \Delta \mathrm{E}=20.19 \mathrm{kcal} / \mathrm{mol}$

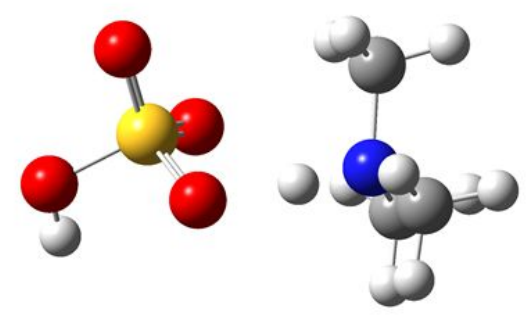

Iso $\mathrm{B}, \Delta \mathrm{E}=1.56 \mathrm{kcal} / \mathrm{mol}$
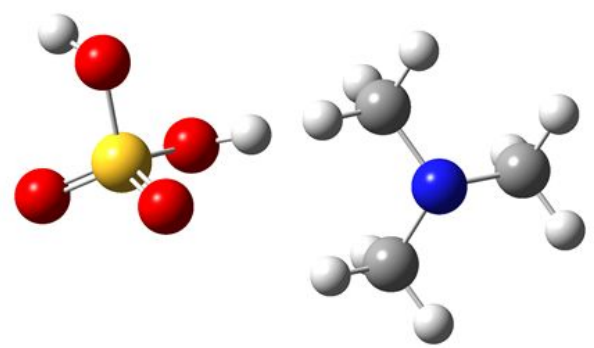

Iso $\mathrm{D}, \Delta \mathrm{E}=20.36 \mathrm{kcal} / \mathrm{mol}$

Figure S7. Optimized geometries of SA-TMA complexes, calculated at the B3LYP-D3(BJ)/6-311++(3df,3pd) level of theory. 
wB97XD/6-311++(3df,3pd)
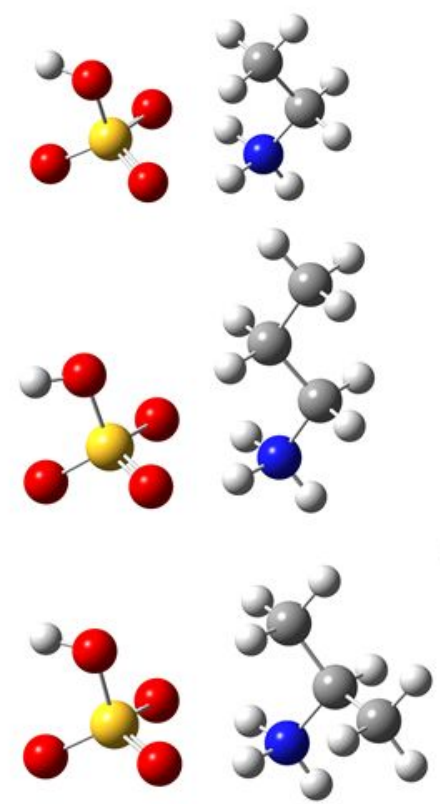

(b) SA-PPA

(a) SA-EA

M06-2X-D3 /6-311++(3df,3pd)
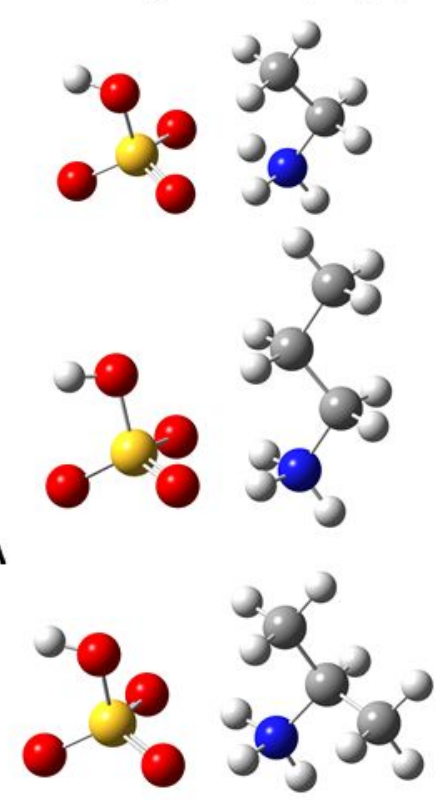

(c) SA-IPA
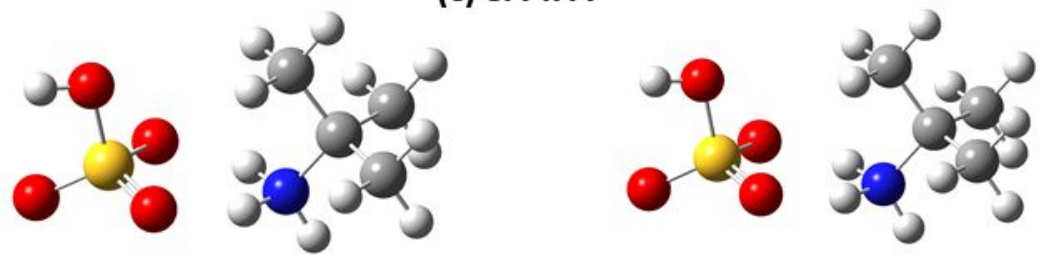

(d) SA-tBA
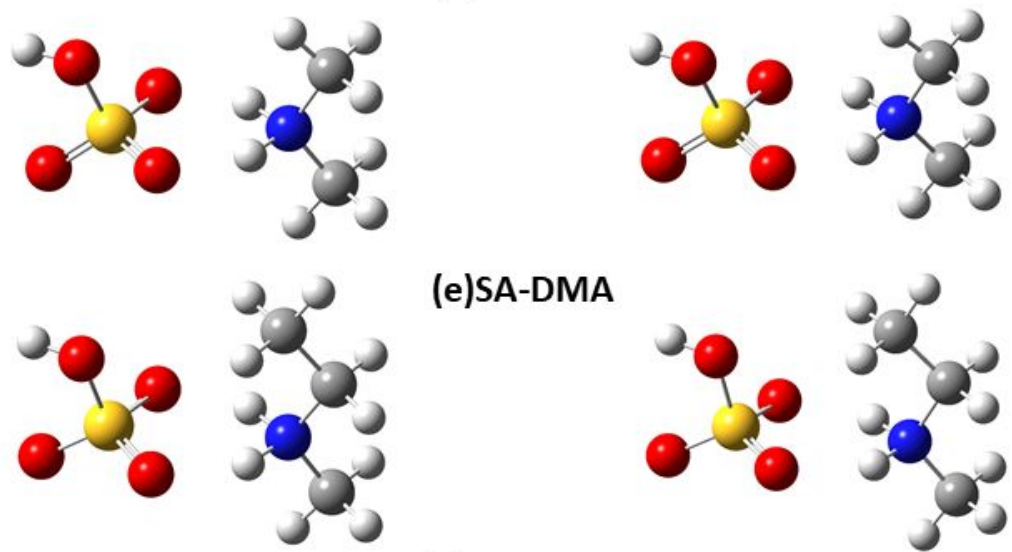

(e)SA-DMA

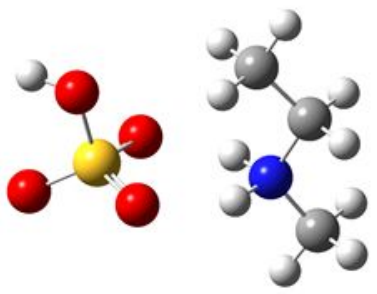

(f) SA-EMA

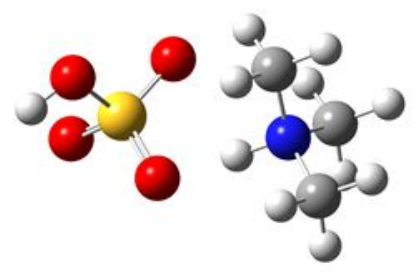

(g) SA-TMA

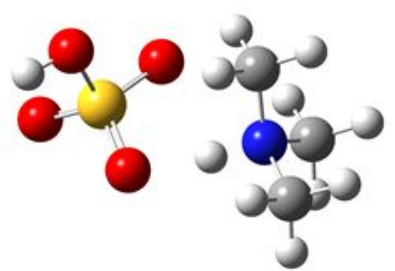

Figure S8. Optimized geometries of sulfuric acid-amine complexes, calculated at the M06-2X-D3/6-311++(3df,3pd) and $\omega$ B97XD/6-311++(3df,3pd) level of 
theory. 
Table S1. Gas-phase basicities and proton affinities of amines. ${ }^{1}$

\begin{tabular}{ccc}
\hline Amine & Gas Basicity $/(\mathrm{kJ} / \mathrm{mol})$ & Proton Affinity $/(\mathrm{kJ} / \mathrm{mol})$ \\
\hline EA & 878.0 & 912.0 \\
PPA & 883.9 & 917.8 \\
IPA & 889.0 & 923.8 \\
tBA & 899.9 & 934.1 \\
DMA & 896.5 & 929.5 \\
EMA & 909.2 & 942.2 \\
TMA & 918.1 & 948.9 \\
\hline
\end{tabular}

Table S2. ZPE-corrected electronic energies, enthalpies, and Gibbs free energy changes for the formation of sulfuric acid-amine complexes at $298.15 \mathrm{~K}$ calculated at the M06-2X-D3/6-311++(3df,3pd) and $\omega \mathrm{B} 97 \mathrm{XD} / 6-311++(3 \mathrm{df}, 3 \mathrm{pd})$ level of theory.

\begin{tabular}{ccccccc}
\hline & \multicolumn{2}{c}{$\Delta \mathrm{E}_{0 \mathrm{~K}}$} & \multicolumn{2}{c}{$\Delta \mathrm{H}_{298 \mathrm{~K}}$} & \multicolumn{2}{c}{$\Delta \mathrm{G}_{298 \mathrm{~K}}$} \\
Complex & \multicolumn{2}{c}{$(\mathrm{kcal} / \mathrm{mol})$} & \multicolumn{2}{c}{$(\mathrm{kcal} / \mathrm{mol})$} & \multicolumn{2}{c}{$(\mathrm{kcal} / \mathrm{mol})$} \\
& M06-2X-D3 & $\omega \mathrm{B} 97 \mathrm{XD}$ & $\mathrm{M} 06-2 \mathrm{X}-\mathrm{D} 3$ & $\omega \mathrm{B} 97 \mathrm{XD}$ & $\mathrm{M} 06-2 \mathrm{X}-\mathrm{D} 3$ & $\omega \mathrm{BB} 97 \mathrm{XD}$ \\
\hline SA-EA & -18.71 & -19.52 & -18.77 & -19.58 & -8.30 & -9.12 \\
SA-PPA & -18.97 & -19.83 & -18.98 & -19.84 & -8.40 & -9.27 \\
SA-IPA & -19.18 & -20.21 & -19.13 & -20.16 & -8.74 & -9.77 \\
SA-tBA & -20.23 & -21.35 & -20.14 & -21.27 & -9.59 & -10.72 \\
SA-DMA & -21.14 & -21.54 & -21.13 & -21.43 & -10.92 & -11.85 \\
SA-EMA & -22.72 & -23.93 & -22.58 & -23.81 & -12.10 & -13.32 \\
SA-TMA & -22.05 & -22.41 & -21.92 & -22.28 & -12.08 & -12.44 \\
\hline
\end{tabular}

\section{References}

(1) Hunter, E. P. L.; Lias, S. G. Evaluated Gas Phase Basicities and Proton Affinities of Molecules: An Update. J. Phys. Chem. Ref. Data 1998, 27, 413-656. 